\title{
A double-reprocessing high-level disinfection protocol does not eliminate positive cultures from the elevators of duodenoscopes
}

\author{
Authors \\ Institutions \\ 1 Division of Gastroenterology-Hepatology, Indiana \\ University, Indianapolis, Indiana, USA \\ 2 Division of Infectious Diseases, Indiana University, \\ Indianapolis, Indiana, USA \\ 3 Department of Pathology and Laboratory Medicine, \\ Division of Clinical Microbiology, Indianapolis, Indiana, \\ USA
}

Douglas K. Rex ${ }^{1}$, Marnie Sieber², Glen A. Lehman', Douglas Webb², Bryan Schmitt ${ }^{3}$, Amy Beth Kressel², Ji Young

Bang ${ }^{1}$, Jeffery Easler ${ }^{1}$, Lee McHenry ${ }^{1}$, Ihab El-Hajj ${ }^{1}$, Evan Fogel' ${ }^{1}$, James Watkins ${ }^{1}$, Stuart Sherman ${ }^{1}$

submitted 23.3.2017

accepted after revision 10.10 .2017

\author{
Bibliography \\ DOI https://doi.org/10.1055/s-0043-122378 \\ Published online: 13.12.2017 | Endoscopy 2018; 50: 588-596 \\ (c) Georg Thieme Verlag KG Stuttgart · New York \\ ISSN 0013-726X
}

Corresponding author

Douglas K. Rex, MD, Indiana University Hospital 4100,

550 N University Blvd, Indianapolis, IN 46202, USA

drex@iu.edu

\section{ABSTRACT}

Background and study aim Duodenoscopes have been the source of serious infection, despite correct performance of high-level disinfection (HLD). This study aimed to observe the impact of performing HLD twice on the rate of positive cultures from duodenoscope elevators.

Methods We performed double HLD (DHLD; i.e. complete manual cleaning followed by automated reprocessing, with the entire process repeated) and then randomly cultured the elevators of our duodenoscopes on about $30 \%$ of occasions.

Results DHLD was associated with positive elevator cultures for any microorganism in $9.4 \%$ of cases, with a $0.8 \%$ rate of known pathogens (627 cultures) between May 2015 and February 2016. After February 2016, and in association with changing the precleaning fluid, as well as use of a new FDA-recommended cleaning brush, the rate of positive cultures for any microorganism after DHLD was $4.8 \%$ and $0.2 \%$ for known pathogens (420 cultures). In a third phase, characterized by a change in personnel performing DHLD and retirement of a duodenoscope with a high rate of positive cultures, the rate of positive cultures for any microorganism was $4.9 \%$ (783 cultures) and the rate of positive culture for known pathogens was $0.3 \%$. To our knowledge, no duodenoscope transmission of infection occurred during the study interval.

Conclusions DHLD resulted in a low rate of positive cultures for known pathogens and for organisms of low pathogenic potential, but did not eliminate these, from duodenoscope elevators. Additional improvements in HLD protocols and/or duodenoscope design are needed.

\section{Introduction}

Duodenoscopes used to perform endoscopic retrograde cholangiopancreatography (ERCP) have structural features that make their successful disinfection more challenging compared with the standard endoscopes that are used for upper gastrointestinal endoscopy and colonoscopy. Specifically, the elevator mechanism of a duodenoscope is accompanied by small spaces that are difficult to access with manual cleaning tools, which are therefore susceptible to the accumulation of debris and bacteria that can potentially transmit infection between patients.

It is recommended that all endoscopes, including duodenoscopes, undergo standardized steps of manual cleansing and disinfection after each use. Failure to reprocess endoscopes ac- cording to these detailed protocols has been recognized for 4 decades to create infection risk [1-12]. Recently, however, a general dogma that endoscopic transmission of infection can be prevented by strict adherence to disinfection protocols has been challenged in the case of duodenoscopes [9-12]. An outbreak of ERCP- transmitted infection could not be attributed to a breakdown in cleaning and disinfection processes [13]. The conclusion that ERCP scopes could transmit infection despite the performance of cleaning and disinfection according to recommended protocols for high-level disinfection (HLD) led to reassessment and revision of HLD recommendations by the Food and Drug Administration (FDA) [14-16] and the Centers for Disease Control and Prevention (CDC) [17].

The urgency to reduce duodenoscope-transmitted infection is magnified because some of the reported outbreaks have 
been with carbapenem-resistant Enterobacteriaceae (CRE) [18, 19], also called carbapenemase-producing Enterobacteriaceae (CPE). CREs are gram-negative bacteria including Klebsiella species, Enterobacter species, and Escherichia coli that hydrolyze carbapenem antibiotics. CRE infections are associated with mortality rates approaching $50 \%$, particularly in immunocompromised patients $[18,19]$.

The FDA has recommended that endoscopy centers perform HLD on duodenoscopes, and that those centers with the capability consider 1 of 4 supplemental measures to reduce infection risk including: microbiological culturing, ethylene oxide (EtO) sterilization, use of a liquid chemical sterilant processing system, or repeat high-level disinfection [20]. In the most recent version of the Multisociety reprocessing guideline [21], it was recommended that units "should review and consider the feasibility and appropriateness for their practice of 1 or more" of these same 4 modalities. Whether any of the supplemental measures recommended by the FDA, or which of them, can eliminate the risk of ERCP-transmitted infection or eliminate positive cultures from duodenoscopes remains uncertain.

Endoscopy centers that have experienced ERCP-transmitted outbreaks of CRE infection have responded in some instances by dramatic changes in their management of duodenoscopes, even prior to the FDA recommendations. These steps have included EtO sterilization of duodenoscopes [22] and universal culturing of duodenoscopes with sequestering of scopes pending culture results [23].

Our center performs approximately 3000 ERCPs per year. Because of an awareness of duodenoscope-transmitted CRE infections without any apparent breakdown in reprocessing [13], we met to develop an approach to preventing transmission of infection by ERCP in our center.

The first issue was the small size of our duodenoscope fleet, which was 16 instruments. It appeared that any measure we would take would require more instruments, and therefore we purchased 40 reprocessed duodenoscopes (8 TJF-Q180V and 32 TJF-160VF; Olympus America Inc., Center Valley, Pennsylvania, USA). Refurbishment of these previously used instruments included inspection of the channels and replacement of any damaged channels.

EtO is available to us, but our capacity to perform EtO sterilization was not sufficient to handle the volume of ERCP procedures performed in our center. Furthermore, EtO creates environmental risks as well as health risks for EtO workers [22, 24, 25 ] and there is uncertainty as to whether EtO damages endoscopes [22,24]. The alternative of universal culture and sequestering of duodenoscopes [23] was also not feasible because our clinical laboratory lacked the capacity to culture the volume of duodenoscopes used in our center, and we could not identify any local environmental laboratory capable of providing universal culturing at a reasonable cost.

We decided to follow another of the recommended courses of action, namely complete doubling of all steps of both manual cleaning and HLD, which we refer to as double HLD (DHLD). DHLD was 1 of the 4 supplemental measures suggested by the FDA, and we devoted resources to culture a feasible fraction of the duodenoscopes after DHLD to monitor the process. Here we report the incidence of positive elevator cultures after DHLD, and the incidence of CRE transmission of infection during application of this program.

\section{Methods}

\section{Study period}

Before initiating the protocol in May 2015, the entire fleet of 56 duodenoscopes (TJF-160VF, $\mathrm{n}=35$; TJF-Q180V, $\mathrm{n}=8$; JF-140, $\mathrm{n}=$ 6; JF-130, $n=3$; TJF-160F, n=2; TJF-140F, n=1; PJF160, $n=1$ ) underwent single-time EtO. The DHLD protocol started in May 2015 and continues through to the present time, with the culture results reported here extending until May 2017.

All culture results were recorded prospectively along with the identity of the duodenoscope and the procedure and culture date. Permission to report the results was granted by our Institutional Review Board.

\section{Double-reprocessing HLD protocol}

Each round of HLD in the DHLD protocol was performed according to the recommendations of the manufacturer, the FDA [14-16], the CDC [17], and the American Society of Gastrointestinal Endoscopy (ASGE) $[26,27]$ that were available or became available during the study period. As per the already established protocol in our unit, all HLD was performed by dedicated staff whose only work in the unit is cleaning and disinfection.

\section{Manual cleaning}

Following each ERCP, the duodenoscope external surface was wiped and the channels were irrigated using an enzymatic solution. From May 2015 through to February 2016, the Compliance EndoKit (Endochoice, Alpharetta, Georgia, USA) was used for this step. In March 2016, after reviewing results, we changed to the Intercept system (Medivators Inc., Minneapolis, Minnesota, USA) for precleaning. In September 2016, the foaming agent in the Intercept system became unavailable and the foaming step was left out of the Intercept precleaning process. From December 2016, Medivators supplied us with a modified foaming agent and we continued the use of this agent through to the end of the study.

After precleaning, the duodenoscope was transferred to the endoscope reprocessing area to complete the manual cleaning process. The duodenoscope was immersed in enzymatic detergent solution (Revital-Ox; Steris Corporation, Mentor, Ohio, USA until March 2016; the Intercept system thereafter) and thoroughly cleaned using a single-use, manufacturer-recommended brush to remove all visible debris. All areas of the duodenoscope were cleaned, including the elevator, elevator recess, elevator locking mechanism, suction port, air/water port, and instrument-channel port. Manual cleaning was continued until no debris was visible on the duodenoscope. The instrument channels and suction port were also irrigated with the detergent solution. The channels were manually cleaned using brushes. Special brushes manufactured by Olympus for cleaning the elevator of the 180 series and subsequently the 160 se- 
ries duodenoscopes were used as they became available (-Fig.1).

\section{Automated reprocessing}

Following manual cleaning, the duodenoscope was placed in an automated endoscope reprocessor (Medivators Inc.) for a total cycle time of approximately 30 minutes (depending on the amount of time taken to heat the water to the appropriate temperature), using a high-level disinfectant (Rapicide PA Parts A and B; Medivators Inc.), then rinsed with filtered sterile water.

The entire process of manual cleaning and reprocessing was then repeated to complete the DHLD protocol. All duodenoscopes were dried thoroughly and hung in the storage closet exposed to room air, except for those selected to undergo culturing. The storage closets did not employ forced air drying. Any duodenoscope not used for 7 days underwent repeat HLD and was then rehung for storage.

\section{Monitoring and culture management}

The infection control department tracked all CRE infections and notified the ERCP physician by electronic alert of any patient undergoing ERCP with a known CRE infection. Any duodenoscope used in a patient with a history of CRE infection underwent HLD and then EtO sterilization. In addition, the medical records of any patient with CRE-positive cultures in the hospital were examined to determine if an ERCP had been performed previously.

Duodenoscopes were not cultured immediately after DHLD. Instead instruments were hung in a storage closet between completion of DHLD and culturing. All duodenoscope cultures were performed by 1 of 5 trained staff in accordance with CDC and FDA guidelines $[14,17]$, with the addition that the channel cultures included material obtained from brushing the channels. For the first 3 months of the DHLD protocol, the most frequently used duodenoscopes in the prior week were randomly selected to undergo culturing of both the working channels and the elevator mechanism. However, after all of the 120 initial cultures from the working channels were found to be negative, the culture process was confined to the elevator mechanism.

We initially cultured 10 , and then increased to 20 , of the most frequently used duodenoscopes per week (10 scopes were cultured every Monday and 10 every Wednesday). This rate of culturing constituted about $30 \%$ of all duodenoscopes when 20 instruments per week were cultured. During this time period, all duodenoscopes in our fleet were cultured at least once. All cultured duodenoscopes underwent a third round of HLD because of the potential risk of endoscope contamination during the culturing process, as per FDA guidelines.

Each swab sample was placed in $10 \mathrm{~mL}$ of tryptic soy broth and vortexed for 5 seconds to dislodge any particulate matter. The swabs were then incubated in tryptic soy broth at $37.0^{\circ} \mathrm{C}$. The broth was checked for turbidity at 24 and 48 hours. If turbidity of the broth was noted, $10 \mu \mathrm{L}$ was subcultured onto tryptic soy agar (TSA) with $5 \%$ sheep blood, MacConkey and CNA agars (Remel, Lenexa, Kansas, USA), and a Gram stain of the broth was performed.

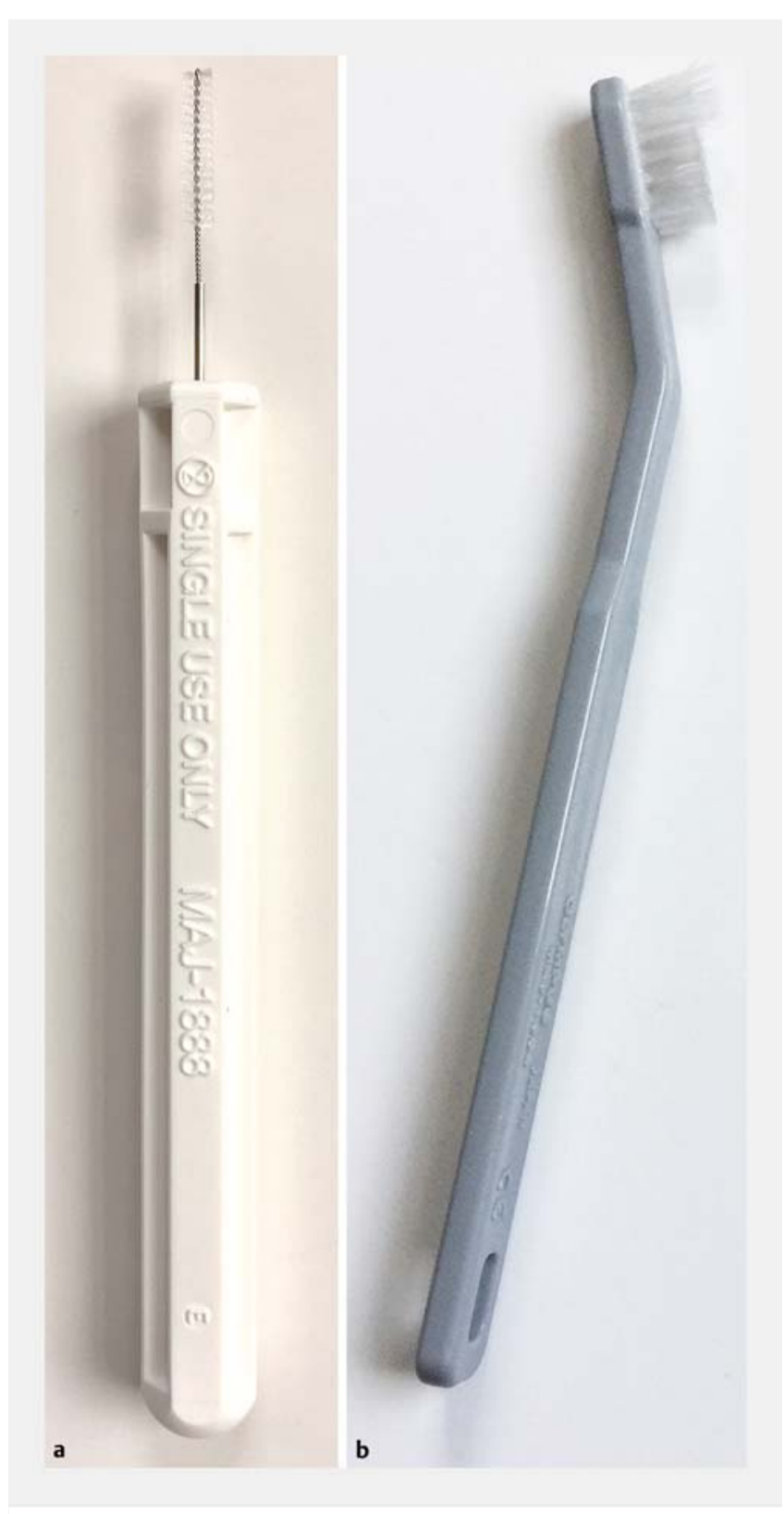

Fig. 1 The Olympus brushes designed for cleaning the elevators of duodenoscopes: a the single-use MAJ-1888 for use on 180 series duodenoscopes, made available in May 2015; $\mathbf{b}$ the reusable MAJ-1534 for use on 160 series duodenoscopes, made available in March 2016.

Each cultured duodenoscope was sequestered pending culture results. Duodenoscopes with negative culture results were released for use after sequestration for 48 hours. Duodenoscopes with positive culture results for any microorganism remained sequestered and underwent repeat culturing followed by another HLD cycle. Those with negative cultures were then released for use, while those with a second consecutive positive culture result for any microorganism underwent EtO sterilization. Duodenoscopes which underwent EtO sterilization were released for use without repeat culturing. A flow diagram of the DHLD protocol is shown in > Fig. 2. 


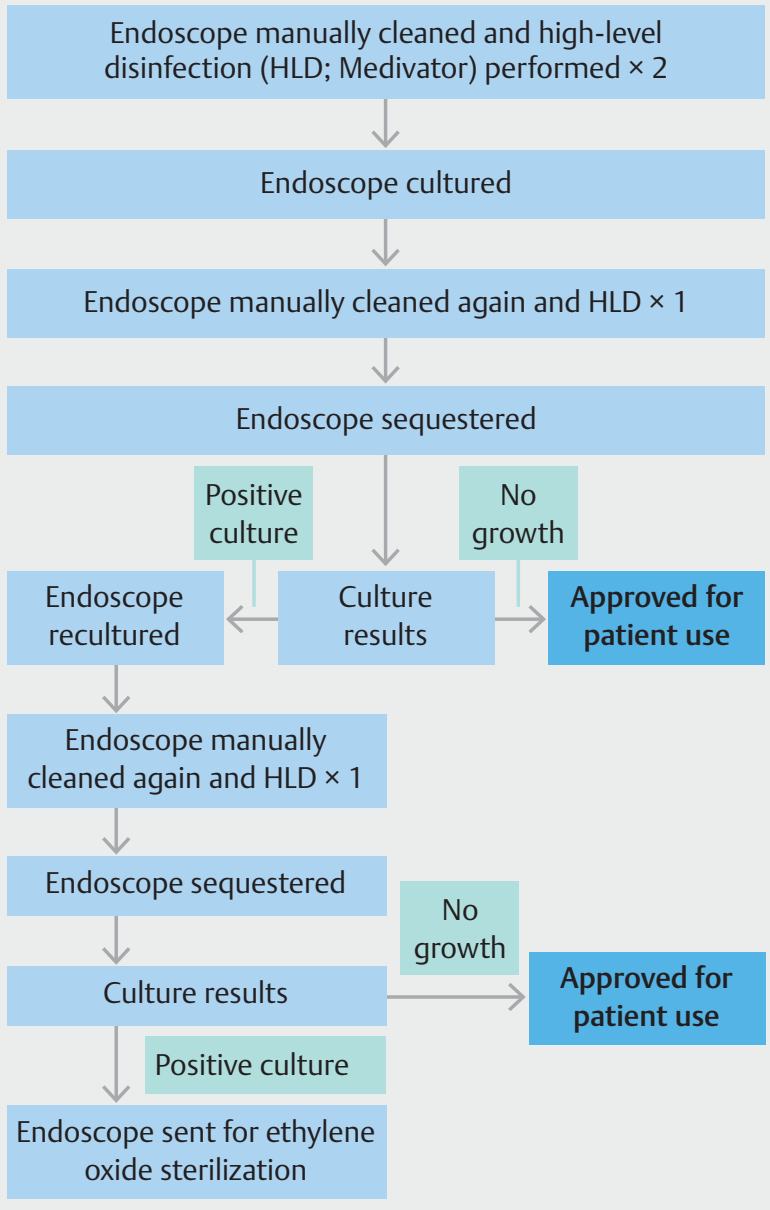

- Fig. 2 Flowchart showing the cleaning and culturing processes for duodenoscopes and the management on the basis of culture results. About $30 \%$ of the duodenoscopes used were cultured.

\section{Study endpoints}

The primary outcome measures were: (i) the incidence of positive cultures (microorganisms with both high and low pathogenic potential) and (ii) the incidence of duodenoscope-associated infection at our institution following implementation of the DHLD protocol. Microorganisms with high pathogenic potential were defined as those known to cause infections of any significance requiring antimicrobial therapy in humans [17], and are also referred to here as "known pathogens." Microorganisms with low pathogenic potential were defined as those not known to cause significant infections in humans or known commensal organisms.

\section{Statistical analysis}

Phase 1

For the initial testing of the DHLD protocol, we performed a sample size calculation using nQuery (Statistical Solutions Ltd, Boston, Massachusetts, USA) with an alpha of 0.05 for a 2 -sided test and a power of $90 \%$ for detecting a $75 \%$ reduction in the rate of positive cultures with known pathogenic organisms following DHLD, compared with the expected rate of $2 \%$ of pathogenic cultures based on an initial report of cultures after singlereprocessing HLD [23]. This resulted in a target sample size of 592 duodenoscope cultures. Duodenoscope culture results were compiled and then summarized as frequencies and proportions using Microsoft Excel (Microsoft Corporation, Redmond, Washington, USA).

\section{Phase 2}

After the sample size of cultures for assessing the DHLD protocol was reached, we elected to test the effect of changing the precleaning solution to the Intercept System. Using a baseline incidence of $9.4 \%$ for any microorganism (see below) determined for the DHLD protocol, we calculated using PASS13 (NCSS, Kaysville, Utah, USA) that a sample size of 263 cultures was needed to determine whether the Intercept system was associated with a $50 \%$ reduction in the overall culture positivity rate.

\section{Phase 3}

In August 2016, we identified that 2 employees involved in the DHLD process may have skipped part or all of the second manual cleaning steps for some of the scopes cleaned during phase 2. When confronted both employees admitted to at least some violations of the protocol and were immediately dismissed from hospital employment. We transferred new personnel in and began their training in DHLD. This event is referred to as the beginning of phase 3 .

We instituted increased unannounced spot audits of the DHLD process to ensure compliance with all steps of the DHLD protocol. Approximately 4 weeks into phase 3 , we stopped using the foaming agent in the Intercept precleaning process because of lack of availability and returned to the previously used standard precleaning measures. An altered foaming agent that is not commercially available was supplied to us 12 weeks later by Medivators; we began using this immediately and continued to use it through to the end of the study. We continued phase 3 until the sample size for the cultures exceeded the target size $(n \geq 263)$.

A chi-squared test was used to compare the occurrence of a positive culture for any microorganism between the scope series (e.g. 180 series vs. 160 series). Fisher's exact test was used to compare the occurrence of a probable pathogen between the scope series.

\section{Results}

From May 2015 to February 2016, 627 cultures were obtained from 56 duodenoscopes reprocessed by DHLD. All duodenoscopes were sampled at least once (the median times each duodenoscope was cultured was 11 [range 1-18]).

From 15 May 2015 to 19 August 2015, none of the 120 nonelevator channel cultures were positive. From 15 May 2015 to 17 February 2016, 59 of 627 elevator cultures (9.4\%) were positive ( $\triangleright$ Table 1$)$. Known pathogenic microorganisms were found in 5 cultures $(0.8 \%)$ from 5 separate cultures ( 1 Candida 
- Table 1 Comparison of high-level disinfection protocols and culture results between 2 studies.

\begin{tabular}{|c|c|c|c|c|}
\hline & \multirow{2}{*}{$\begin{array}{l}\text { Ross et al. } \\
\text { [23] }\end{array}$} & \multicolumn{3}{|l|}{ Current study } \\
\hline & & Phase 1 & Phase 2 & Phase 3 \\
\hline Total number of duodenoscopes cultured, $\mathrm{n}$ & 1524 & 627 & 420 & 783 \\
\hline Overall culture positive rate, $n(\%)$ [95\%Cl] & $200(13.1)$ & $59(9.4)[7.2-12.0]$ & $20(4.8)[2.9-7.3]$ & $38(4.9)[3.5-6.6]$ \\
\hline Pathogenic culture positive rate, $\mathrm{n}(\%)[95 \% \mathrm{Cl}]$ & $29(1.9)$ & $5(0.8)[0.3-1.9]$ & $1(0.2)[0.0-1.3]$ & $2(0.3)[0.0-0.9]$ \\
\hline Non-pathogenic culture positive rate, $\mathrm{n}(\%)[95 \% \mathrm{Cl}]$ & $171(11.1)$ & $54(8.6)[6.5-11.1]$ & $19(4.5)[2.8-7.0]$ & $36(4.6)[3.2-6.3]$ \\
\hline Number of sessions of manual cleaning & 1 & 2 & $2^{1}$ & 2 \\
\hline Number of sessions of automated reprocessing & 1 & 2 & 2 & 2 \\
\hline Percentage of duodenoscopes cultured & 100 & $\sim 30$ & $\sim 30$ & $\sim 30$ \\
\hline
\end{tabular}

- Table 2 Summary of duodenoscope culture results in the 3 phases of the study.

\begin{tabular}{|c|c|c|c|}
\hline & Phase 1 & Phase 2 & Phase 3 \\
\hline Total cultures & 627 & 420 & 783 \\
\hline Total positive cultures, $\mathrm{n}(\%)[95 \% \mathrm{Cl}]$ & $59(9.4)[7.2-12.0]$ & $20(4.8)[2.9-7.3]$ & $38(4.9)[3.5-6.6]$ \\
\hline \multicolumn{4}{|c|}{ Organisms of high pathogenic potential (“known pathogens”), n (\%) [95\%Cl] } \\
\hline - Overall & $5(0.8)[0.3-1.9]$ & $1(0.2)[0.0-1.3]$ & $2(0.3)[0.0-0.9]$ \\
\hline - Enterococcus & $3(0.5)[0.1-1.4]$ & $1(0.2)[0.0-1.3]$ & \\
\hline - Candida species & $1(0.16)[0.0-0.9]$ & $0(0)[0.0-0.9]$ & $2(0.3)[0.0-0.9]$ \\
\hline - Zygomycete & $1(0.16)[0.0-0.9]$ & $0(0)[0.0-0.9]$ & $0(0)[0.0-0.5]$ \\
\hline \multicolumn{4}{|c|}{ Organisms of low pathogenic potential, n (\%) $[95 \% \mathrm{Cl}]$} \\
\hline - Overall & $54(8.6)[6.5-11.1]$ & $19(4.5)[2.8-7.0]$ & $36(4.6)[3.2-6.3]$ \\
\hline - Micrococcus & $40(6.4)[4.6-8.6]$ & $6(1.4)[0.5-3.1]$ & $7(0.9)[0.4-1.8]$ \\
\hline - Coagulase-negative Staphylococcus & $10(1.6)[0.8-2.9]$ & $5(1.2)[0.4-2.8]$ & $24(3.1)[2.0-4.5]$ \\
\hline - Bacillus & $4(0.6)[0.2-1.6]$ & $6(1.4)[0.5-3.1]$ & $4(0.5)[0.1-1.3]$ \\
\hline - Corynebacterium & $0(0)[0.0-0.6]$ & $2(0.5)[0.1-1.7]$ & $1(<0.1)[0.0-0.7]$ \\
\hline
\end{tabular}

glabrata, 1 Zygomycete and 3 Enterococcus spp.) (\ Table 2). The remaining positive cultures consisted of microorganisms of low pathogenic potential and included Micrococcus spp. in 40, coagulase-negative Staphylococcus spp. in 10, and Bacillus spp. in 4 ( $\triangleright$ Table 2 ).

6 duodenoscopes had 2 consecutive positive cultures (with any microorganism) and by protocol underwent EtO sterilization. A single 160 series duodenoscope did not have 2 consecutive positive cultures but had an overall $25 \%$ rate of positive cultures. This same instrument accounted for 2 of the initial 6 positive cultures for known pathogens (including the 1 culture of a known pathogen in the second phase of the study-see below). This duodenoscope underwent EtO followed by culture after every use. Culture after every use quickly identified that the duodenoscope could not be effectively disinfected by DHLD and it was removed from service near the end of phase 2 (see below).

In February 2016, after a final review of the culture results noted above, we changed the precleaning solution to the Intercept system (Medivators Inc.), initiating the period we referred to as phase 2 . We continued the culture process throughout phase 2, but allowed a 2-week run-in period of performing DHLD with the Intercept system before measuring the culture rate associated with the Intercept system. Between March and August 2016, 420 duodenoscope elevator cultures were obtained, with 20 positive cultures (4.8\%) for any microorganism and $1(0.2 \%)$ for a known pathogen. The known pathogen was again an Enterococcus species, and the organisms of low pathogenic potential were again predominantly coagulase-nega- 
- Table 3 Rates of culture-positive duodenoscopes among the 4 different series of Olympus instruments in our fleet for the 1830 cultures in the entire study.

\begin{tabular}{|l|c|l|l|}
\hline $\begin{array}{l}\text { Olym- } \\
\text { pus } \\
\text { series }\end{array}$ & $\begin{array}{l}\text { Number } \\
\text { of cul- } \\
\text { tures } \\
\text { taken }\end{array}$ & $\begin{array}{l}\text { Total positive } \\
\text { cultures for any } \\
\text { microorganism, } \\
\mathbf{n}(\%)[95 \% \mathrm{CI}]\end{array}$ & $\begin{array}{l}\text { Number of positive } \\
\text { cultures for known } \\
\text { pathogens, } \mathbf{n}(\%) \\
{[95 \% \mathrm{CI}]}\end{array}$ \\
\hline 180 & 269 & $13(4.8)[2.6-8.1]$ & $0(0)[0.0-1.4]$ \\
\hline 160 & 1291 & $87(6.7)[5.4-8.3]$ & $5(0.4)[0.1-0.9]$ \\
\hline 140 & 186 & $10(5.4)[2.6-9.7]$ & $3(1.6)[0.3-4.6]$ \\
\hline 130 & 84 & $7(8.3)[3.4-16.4]$ & $0(0)[0.0-4.3]$ \\
\hline Cl, confidence interval. & & \\
\hline
\end{tabular}

tive Staphylococcus spp., Bacillus spp., and Micrococcus spp. ( Table 2).

Phase 3 of the culture process (August 2016 to May 2017) was characterized by an abrupt change in personnel performing the DHLD protocol and also initially, beginning about 4 weeks into phase 3 , by a lack of availability of the foaming agent in the Intercept precleaning protocol. During phase 3, the rate of positive cultures for any microorganism was $4.9 \%$ and the rate for known pathogens was $0.3 \%$ (783 cultures). During phase 3 , there appeared to be a spike in the positive culture rate during the first 4 weeks (during which time the 2 new personnel were being trained) with an overall rate of positive cultures for any microorganism of $12.7 \%$ and for pathogens of $1.3 \%$ (79 cultures). In the final 704 cultures of phase 3 , the rate of positive cultures for any microorganism was 3.9\% and the rate of culture for known pathogens was $0.14 \%$. During phases 2 and 3 combined, 7 more duodenoscopes had 2 consecutive positive cultures for any micoorganism and underwent EtO sterilization.

- Table 3 shows the overall number of positive cultures according to the Olympus series of duodenoscopes and demonstrates that the rate of positive cultures was similar for all series. There was no difference between the 4 series of duodenoscopes in the frequency of positive cultures for any microorganism $(P=0.53)$. Similarly, there was no difference between the duodenoscope series in the frequency of positive cultures for probable pathogens $(P=0.13)$. Also, there was no difference between the 180 duodenoscopes and the other 3 series combined for the frequency of positive cultures for any microorganism $(P=0.26)$ or for probable pathogens $(P=0.61)$. 3 of the 5 positive cultures for pathogens from 160 series duodenoscopes came from 3 different refurbished 160 series instruments.

Of the 13 instruments for which there were 2 positive cultures in succession, the organism was the same genus in both positive cultures for 8 instruments. We did not further subtype the organisms or match them for antimicrobial sensitivity to prove the organisms were identical in the 8 noted cases.

During the study interval, our infection control monitors identified 17 patients in the hospital with new positive CRE cultures. The number of patients admitted to the hospital with pre-existing CRE infection is uncertain. There were no duode- noscope-associated CRE infections identified during the study period.

\section{Discussion}

Protection of patients from iatrogenic injury is of fundamental and particular importance to the practice of medicine. Recognition of the potential of ERCP to transmit life-threatening infections, despite correct performance of all recommended duodenoscope reprocessing steps, is of great concern to the public, to endoscopists, to gastroenterology and endoscopy professional societies, and to regulatory agencies.

Awareness of this issue raises particular concerns at an institution like ours, where the high volume of ERCPs places a large number of patients at potential risk of serious infection. We considered that the high volume of ERCP procedures performed at our institution placed a special burden on us to develop an organized and dedicated response to protect our patients. The nature of that response is partly dictated by the feasibility of potential protective measures, as it is in any institution seeking to respond to the threat of duodenoscope transmission of CRE infection. In our institution we elected to evaluate a program of DHLD of duodenoscopes accompanied by culture and sequestering of a subset of instruments.

This observational report describes the results of our DHLD protocol. We did not observe CRE infections transmitted by ERCP during the study period. However, our entire study was uncontrolled and the expected rate of CRE infections by ERCP in a similar period in our institution is unknown. Furthermore, duodenoscope-associated infections are notoriously under-reported, especially in tertiary referral centers with a long-distance referral base. Contaminated duodenoscopes can also result in colonization of patients with CREs, and CRE infections can present weeks to months after the inciting ERCP. Therefore, the culture results after DHLD are also informative.

The primary result of our study was that DHLD was associated with low rates of positive culture for both commensals (low pathogenic potential) and known pathogens (high pathogenic potential) but did not eliminate positive cultures. We had a steady flow of positive cultures throughout the study. All 3 phases of our study were of substantial size and, although we altered the DHLD protocol in an effort to improve the results, all 3 phases had significant rates of positive culture. Therefore, our primary result is that DHLD does not eliminate positive cultures from the elevators of duodenoscopes. Our results suggest that the FDA [20] and the Multisociety guideline [21] should review their suggestion to utilize DHLD if feasible.

The significance of culturing non-pathogenic organisms from the elevator mechanism is uncertain, but it seems reasonable to be concerned that even culture of non-pathogenic organisms could be a signal pointing toward ineffective cleaning or disinfection of the elevator mechanism. It remains uncertain whether the goal of clinical trials or clinical practice should be the elimination of culture positivity for pathogens or for any microorganism.

In any case, as we did not culture all of the duodenoscopes, it seems clear that in some instances we must have performed 
ERCPs with duodenoscopes that had elevators contaminated with pathogens. Given this, our preference would be to move to a policy of universal culture and continue that policy until a reliable reprocessing method that eliminates pathogens from the elevator region of duodenoscopes becomes available. However, we have not yet identified an economically feasible method to perform universal culture of our duodenoscopes, either within our clinical laboratory or using an external environmental laboratory. These approaches might be more feasible for an ERCP program with a smaller volume of cases or a larger clinical laboratory.

At this time, having concluded that DHLD cannot effectively eliminate positive cultures from duodenoscope elevators, we plan a controlled trial of cold sterilization (peracetic acid) vs. DHLD accompanied by continuation of the current culture protocol. Table 1 shows the uncontrolled comparison of positive culture rates after HLD at Virginia Mason [22] and in the 3 phases of our study.

After an initial period of observation of DHLD, we intentionally changed the precleaning solution to the Medivators Intercept product and then continued the monitoring process. Medivators had presented some unpublished evidence to us that their Intercept product could reduce biofilm on colonoscopes. This change was associated with a reduction in the rate of positive cultures of any microorganism from $9.4 \%$ to $4.2 \%$, and of any known pathogen from $0.8 \%$ to $0.3 \%$. However, this reduction in the rate is a non-randomized observation, and the change in product coincided roughly in time with the introduction by Olympus of a new cleaning brush for 160 series duodenoscopes, and a substantial portion of our fleet is made up of 160 series duodenoscopes. Our results suggest that the foaming agent warrants a controlled assessment of its effect on positive culture rates. Furthermore, our results may be of use in formulating hypotheses and sample size estimates for better controlled prospective trials of cleaning methods and agents.

We also identified that some endoscopes in phase 2 did not undergo the full second manual disinfection process, though we believe that the first manual disinfection and both rounds in the automatic reprocessor occurred in all cases. This event highlights the importance of monitoring and auditing the HLD or DHLD process, which remains subject to human error. We did not see a further drop in the rate of positive cultures in phase 3 , despite retiring 1 duodenoscope with an excessive number of positive cultures and increasing unannounced spot audits of the DHLD process (which did not demonstrate any breaches of the DHLD protocol in phase 3). As noted above, none of the measures we employed in any of the 3 phases eliminated positive cultures from duodenoscope elevators.

When the problem of duodenoscope transmission of CRE was initially recognized, it appeared the issue might be peculiar to Olympus 180 series instruments [16]. Our results show clearly that the issue also pertains to 160 series and older instruments. Available evidence indicates that the issue pertains to duodenoscopes regardless of manufacturer [1-12]. We use our DHLD protocol for cleaning of endoscopic ultrasound (EUS) scopes, which also have elevators, though there is cur- rently uncertainty about the potential of EUS scopes to transmit infection.

Our study strongly supports a policy of systematic culture to identify individual duodenoscopes that are recurrently culture positive, which either require special treatment and monitoring, or removal from service. 1 single instrument accounted for 2 of the 6 positive cultures for pathogens observed during phases 1 and 2, and had a $25 \%$ rate of overall positive cultures. This observation raises concerns about increased infection risk in ERCP programs with no biological monitoring of duodenoscopes, because 1 or more instruments that are prone to contamination might go unrecognized for prolonged intervals, thereby potentially causing multiple infections.

We identified 12 instances in which a duodenoscope elevator was culture positive on 2 successive cultures. In 8 of these cases the organism was the same genus on both occasions, so may have represented persistent colonization of the elevator mechanism. However, we did not further subtype the organisms or match them by antimicrobial sensitivities to determine with confidence that the organism was the same on both occasions. In at least 4 cases, different organisms on the 2 positive cultures suggests that environmental contamination might have contributed to some of the positive cultures in our study. Certainly, the interpretation of positive cultures for commensal organisms is subject to error, as these organisms are potentially introduced by the culture process rather than instrument contamination by exposure to patients.

Our study has a number of limitations. First, we did not randomize duodenoscopes to DHLD vs. single-processing. Instead, in phase 1 , we compared post-DHLD positive culture rates to the rates after single reprocessing at a different institution. Primarily we used the rates of positive culture from this other institution to calculate a sample size for the number of cultures needed in the first portion of this study. However, we did not consider a comparison between culture rates at our institution and another institution to be a controlled experiment.

Since we initiated our own study, Brandabur et al. [28] reported the results of single HLD followed by culture of the elevator and/or working channels of duodenoscopes and linear echoendoscopes at 21 centers. In 4032 surveillance cultures from 2238 instruments, $8.4 \%$ of instruments had positive cultures for any organism and $0.9 \%$ had positive cultures for "organisms of high concern." The exact rate of positive cultures from elevators overall in the study was unclear, but in 12 instruments with a positive culture of a pathogen in which the source of the culture was clear, all 12 instruments had positive cultures from the elevator. Therefore, the rate of positive culture for pathogens from the elevator after single HLD in the study was likely higher than we observed after DHLD. Again, however, uncontrolled comparisons of culture positivity rates between different studies is potentially misleading. We therefore consider our report to be only an observational study describing what might be expected with DHLD in a prospective experience in a tertiary center. Given this limitation we certainly cannot exclude that DHLD might reduce the risk of positive cultures and infection relative to HLD. However, our results clearly indicate 
that DHLD does not eliminate the risk of positive cultures for pathogenic organisms.

As a second limitation of our study, in the second phase of the study, we did not randomize instruments to cleaning with Intercept vs. any other product. The limitations created by this design have been noted above. In general, the non-randomized design and the multiple changes to the DHLD protocol made between and during phases 2 and 3 makes the interpretation of culture rates between phases challenging. Instead, we believe the persistence of positive cultures despite multiple changes in the protocol is the main result of this study.

Third, we cultured only about $30 \%$ of the duodenoscopes treated by DHLD, so we could have missed cultures of serious pathogens including CREs. However, our sample size of cultures is large and probably fairly represents the rate of positive culture that would be observed with universal culturing after DHLD. There were additional limitations related to our culture process. For example, we did not culture scopes immediately after DHLD, which might have reduced our rate of positive culture, if there was additional drying of the biofilm. Also, we did not culture instruments after EtO, and EtO may not sterilize instruments when biofilm is present.

At this juncture, our goal is to perform a controlled trial of cold sterilization at our center. Of the options suggested by the FDA and the Multisociety guideline [20,21], liquid chemical sterilization is the remaining option that, given the large volume of ERCPs at our center, currently seems most feasible for us to implement with the goal of eliminating positive elevator cultures. Whether liquid chemical sterilization can eliminate positive cultures is uncertain [28], but has not been fully evaluated.

For the reasons stated earlier, universal culturing [23] is not feasible at our center and EtO sterilization is neither feasible nor desirable [22, 24, 25]. HLD remains an important approach for further investigation and improvement because its cost-effectiveness is superior to other measures [29]. When HLD or DHLD are used at the current time, our results indicate they should be accompanied by a degree of microbiological monitoring, at least until the design of the elevator is corrected to allow consistent effective HLD.

We emphasize that feasibility dominated our approach to disinfection during the study period. Therefore, we did not culture all of our duodenoscopes after each use because our clinical laboratory found universal culture to be infeasible. We utilized EtO only when instruments were used on patients with documented infection with a multidrug-resistant organism, or when cultures from a particular instrument were positive twice in succession, because we did not have facilities available to perform EtO on all of our instruments. We suspect that many endoscopy units would encounter similar feasibility issues with applying 1 or all of the FDA suggested extra disinfection steps on a universal basis. We realize that any approach that is not applied universally to all patients is suboptimal. These challenges in feasibility underscore the need to develop a novel approach to disinfection or sterilization of duodenoscopes that is both universally effective and widely feasible.
In summary, DHLD did not eliminate the risk of transmission of serious infection by duodenoscopes. DHLD, although suggested by both the FDA and the Multisociety guideline as a potential extended reprocessing step for units with the feasibility to conduct it, is not the answer to eliminating positive cultures from duodenoscopes. Our results indicate the need for further modifications of HLD and/or duodenoscope design to achieve eradication of organisms from the elevators of duodenoscopes.

\section{Competing interests}

Douglas K. Rex is a consultant for Olympus Corporation.

\section{References}

[1] Classen DC, Jacobson JA, Burke JP et al. Serious Pseudomonas infections associated with endoscopic retrograde cholangiopancreatography. Am J Med 1988; 84: 590-596

[2] Earnshaw JJ, Clark AW, Thom BT. Outbreak of Pseudomonas aeruginosa following endoscopic retrograde cholangiopancreatography. J Hosp Infect 1985; 6: 95-97

[3] Kovaleva J, Peters FT, van der Mei HC et al. Transmission of infection by flexible gastrointestinal endoscopy and bronchoscopy. Clin Microbiol Rev 2013; 26: $231-254$

[4] Low DE, Micflikier AB, Kennedy JK et al. Infectious complications of endoscopic retrograde cholangiopancreatography. A prospective assessment. Arch Intern Med 1980; 140: 1076-1077

[5] Seoane-Vazquez E, Rodriguez-Monguio R, Visaria J et al. Exogenous endoscopy-related infections, pseudo-infections, and toxic reactions: clinical and economic burden. Curr Med Res Opin 2006; 22: 2007 2021

[6] Struelens M], Rost F, Deplano A et al. Pseudomonas aeruginosa and Enterobacteriaceae bacteremia after biliary endoscopy: an outbreak investigation using DNA macrorestriction analysis. Am J Gastroenterol 1993; 95: 489-498

[7] Allen JI, Allen MO, Olson MM et al. Pseudomonas infection of the biliary system resulting from use of a contaminated endoscope. Gastroenterology 1987; 92: 759-763

[8] Schousboe M, Carter A, Sheppard PS. Endoscopic retrograde cholangio-pancreatography: related nosocomial infections. N Z Med J 1980; 92: $275-277$

[9] Alrabaa SF, Nguyen P, Sanderson R et al. Early identification and control of carbapenemase-producing Klebsiella pneumoniae, originating from contaminated endoscopic equipment. Am J Infect Control 2013; 41: $562-564$

[10] Aumeran C, Poincloux L, Souweine B et al. Multidrug-resistant Klebsiella pneumoniae outbreak after endoscopic retrograde cholangiopancreatography. Endoscopy 2010; 42: 895-899

[11] Carbonne A, Thiolet JM, Fournier $S$ et al. Control of a multi-hospital outbreak of KPC-producing Klebsiella pneumoniae type 2 in France, September to October 2009. Euro Surveill 2010: 15

[12] Gastmeier P, Vonberg RP. Klebsiella spp. in endoscopy-associated infections: we may only be seeing the tip of the iceberg. Infection 2014; 42: $15-21$

[13] Epstein L, Hunter JC, Arwady MA et al. New Delhi metallo-beta-lactamase-producing carbapenem-resistant Escherichia coli associated with exposure to duodenoscopes. JAMA 2014; 312: 1447-1455

[14] US FDA. Infections associated with reprocessed duodenoscopes. 2015: Available from: https://wayback.archive-it.org/7993/ 
20161022055601/http://www.fda.gov/MedicalDevices/ProductsandMedicalProcedures/ReprocessingofReusableMedicalDevices/ ucm454630.htm [Accessed: 19 October 2017]

[15] US FDA. Endoscopic retrograde cholangiopancreatography (ERCP) duodenoscopes: FDA Safety Communication - Design may impede effective cleaning. 2015: Available from: https://www.fda.gov/safety/medwatch/safetyinformation/safetyalertsforhumanmedicalproducts/ucm434922.htm [Accessed: 19 October 2017]

[16] US FDA. Olympus validates new reprocessing instructions for model TJF-Q180V duodenoscopes: FDA Safety Communication. 2015: Available from: https://wayback.archive-it.org/7993/ 20161022044047/http://www.fda.gov/MedicalDevices/Safety/AlertsandNotices/ucm439999.htm [Accessed: 19 October 2017]

[17] CDC. Interim Duodenoscope Surveillance Protocol. 2015: Available from: https://www.cdc.gov/hai/organisms/cre/cre-duodenoscopesurveillance-protocol.html [Accessed: 19 October 2017]

[18] Guh AY, Bulens SN, Mu Y et al. Epidemiology of carbapenem-resistant Enterobacteriaceae in 7 US Communities, 2012-2013. JAMA 2015; 314: $1479-1487$

[19] Patel G, Huprikar S, Factor SH et al. Outcomes of carbapenem-resistant Klebsiella pneumoniae infection and the impact of antimicrobial and adjunctive therapies. Infect Control Hosp Epidemiol 2008; 29: 1099-1106

[20] US FDA. Duodenoscope reprocessing: FDA Safety Communication Supplemental measures to enhance reprocessing. 2015: Available from: https://www.fda.gov/Safety/MedWatch/Safetylnformation/ SafetyAlertsforHumanMedicalProducts/ucm457132.htm [Accessed: 19 October 2017]
[21] Petersen BT, Cohen J, Hambrick RD 3rd et al. Multisociety guideline on reprocessing flexible Gl endoscopes: 2016 update. Gastrointest Endosc doi:10.1016/j.gie.2016.10.002

[22] Smith ZL, Oh YS, Saeian K et al. Transmission of carbapenem-resistant Enterobacteriaceae during ERCP: time to revisit the current reprocessing guidelines. Gastrointest Endosc 2015; 81: 1041 - 1045

[23] Ross AS, Baliga C, Verma P et al. A quarantine process for the resolution of duodenoscope-associated transmission of multidrug-resistant Escherichia coli. Gastrointest Endosc 2015; 82: 477-483

[24] Humphries RM, McDonnell G. Superbugs on duodenoscopes: the challenge of cleaning and disinfection of reusable devices. J Clin Microbiol 2015; 53: 3118-3125

[25] Haney PE, Raymond BA, Lewis LC. Ethylene oxide. An occupational health hazard for hospital workers. AORN J 2015; 51: 480-481, 483, 485-486

[26] ASGE Quality Assurance in Endoscopy Committee, Petersen BT, Chennat J et al. Multisociety guideline on reprocessing flexible gastrointestinal endoscopes: 2011. Gastrointest Endosc 2011; 73: $1075-1084$

[27] ASGE Technology Committee, Varadarajulu S, Banerjee $S$ et al. Gl endoscopes. Gastrointest Endosc 2011; 74: 1-6 e6

[28] Brandabur JJ, Leggett JE, Wang L et al. Surveillance of guideline practices for duodenoscope and linear echoendoscope reprocessing in a large healthcare system. Gastrointest Endosc 2016; 84: 392 - 399

[29] Almario CV, May FP, Shaheen N] et al. Cost utility of competing strategies to prevent endoscopic transmission of carbapenem-resistant Enterobacteriaceae. American J Gastroenterol 2015; 110: 1666 - 1674 